\title{
Changes in Cytokine Production During Pregnancy in Patients with Graves' Disease
}

\author{
Brian M. Jones, ${ }^{1}$ Janette S.Y. Kwok, ${ }^{1}$ and Annie W.C. Kung ${ }^{2}$
}

In order to investigate the role of type 1 and type 2 cytokines in the remission of Graves' disease (GD) during pregnancy, spontaneous and mitogen-stimulated production of interleukin (IL)-4, IL-6, IL-10, IL-12, interferon- $\gamma$ $(\mathrm{IFN}-\gamma)$, and tumour necrosis factor- $\alpha$ (TNF- $\alpha)$ were measured by enzyme-linked immunospot assay of peripheral blood mononuclear cells from 10 pregnant women with GD, 8 healthy pregnant women, and 10 healthy nonpregnant women. Tests were performed in the first, second, and third trimesters of pregnancy and 10-17 weeks after delivery. IL-4 production was not affected greatly by normal or GD pregnancy, whereas IFN- $\gamma$ production was suppressed throughout pregnancy but returned to normal levels after delivery in both controls and patients. IL-6 and TNF- $\alpha$ tended to be higher in GD pregnancy than normal pregnancy, especially in the second and third trimesters. Controls had raised IL-10 in the first trimester with a return to normal levels by the third trimester, whereas patients had raised levels throughout pregnancy. IL-12 levels were suppressed to a greater extent in control than Graves' pregnancy, especially during the second and third trimesters. Ratios of IL10:IL12 in phytohemaglutinin (PHA)-stimulated cultures were much lower in GD than normal pregnancy and cross-regulation of IL-10 and IL-12 may be deficient in GD.

\section{Introduction}

$\mathbf{N}$ ORMAL PREGNANCY is associated with development of a type 2 polarized cytokine profile that serves to suppress the maternal cell-mediated antifetal immune response $(1,2)$. Marked increases in the levels of cortisol, estrogen, and progesterone that occur during pregnancy appear to mediate this phenomenon; glucocorticoids inhibit interleukin (IL)12 but not IL-10 production (3), estradiol enhances antigenstimulated IL-10 production in vitro (4) and progesterone induces and enhances IL-4 production by human T cells (5).

The clinical activity of autoimmune disease frequently changes during pregnancy. Rheumatoid arthritis, an autoimmune disease driven by increased production of proinflammatory tumour necrosis factor- $\alpha$ (TNF- $\alpha$ ) (6), becomes less active during pregnancy and flares postpartum (7), presumably because of increased levels of anti-inflammatory IL10. In contrast the influence of pregnancy on disease activity in women with type 2 cytokine-driven systemic lupus erythematosus is variable, with exacerbation due to increased autoantibody and immune complex formation (8) occurring in at least $13 \%$ of patients (9).

There is conflicting evidence concerning cytokine polarization in Graves' disease (GD). Intrathyroidal lymphocytes had increased expression of IL-2, IL-4, IL-10 and interferon- $\gamma($ IFN- $\gamma)(10)$, although large activated T cells, presumably responsible for autoimmune damage, produced predominantly IL-4 (11). IL-2, IFN- $\gamma$, TNF- $\alpha$, and IL-10 but not IL-4, messenger ribonucleic acid (mRNA) were found in intrathyroidal lymphocytes from GD patients in one study (12), while another showed increased mRNA for IL-4 and IL-10 in thyroid biopsies from GD patients with high levels of antithyroid antibodies (13). A recent study demonstrated complementary DNA for IL-4 and IL-10, but not IFN- $\gamma$, in T cells cloned from GD thyroid tissue (14).

Phytohemagglutinin (PHA)-stimulated peripheral blood mononuclear cells (PBMC) from untreated GD patients produced more IL-4 than normal controls (15) and ratios of IFN$\gamma$ or TNF- $\alpha$ to IL- 4 or IL-10 were lower in GD patients than controls (16). An enzyme-linked immunospot (ELISPOT) assay revealed significantly reduced IL-4 and INF- $\gamma$-secreting PBM, but normal IL-6, IL-10, IL-12, and TNF- $\alpha$ production before treatment of GD patients with radioactive iodine, and prolonged normalization of IFN- $\gamma$ but not IL- 4 after therapy (17). Although the evidence is somewhat conflicting, GD appears to be driven by type 2 cytokines and because IFN- $\gamma$, IL-2, and TNF- $\alpha$ can suppress production of antithyroid antibodies by thyroid B-cells in vitro (18), GD also appears to be regulated by type 1 cytokines. It is therefore surprising that GD shows features of type 1 cytokine-driven autoim- 
mune diseases during pregnancy, tending to remit during the second and third trimesters and to recur after delivery (19). This is partially explained by a pregnancy induced change from stimulatory to blocking autoantibody activity (20). The studies described here indicate that there are differences between pregnancy induced cytokine changes in normal subjects and GD patients, most notably impaired cross-regulation of IL12 by IL-10.

\section{Materials and Methods}

\section{Experimental subjects}

Diagnosis of GD was based on the presence of soft diffuse goiter, raised serum free thyroxine $\left(\mathrm{T}_{4}\right)$ and suppressed thyroid stimulating hormone (TSH). Ten pregnant GD patients ages 30-39 (mean $32.5 \pm 3.1$ ) years were studied for cytokine production at 10-14, 21-24, and 31-35 weeks gestation and at 10-12 weeks postpartum. Patients were receiving less than $10 \mathrm{mg}$ of carbimazole or less than $100 \mathrm{mg}$ of propylthiouracil daily for the 6 months preceding conception and during pregnancy they were given the lowest dose of drugs that could maintain euthyroidism. All drugs were stopped by the third trimester.

Eight healthy control pregnant women ages 30-37 (32.8 \pm 2.4) years were studied at 11-14, 21-24, and 30-33 weeks gestation and at 11-17 weeks after delivery. Ten healthy nonpregnant females ages 29-43 (35.8 \pm 4.9$)$ were also studied. All subjects were of Chinese ethnic origin and gave informed consent. The study was approved by the Ethics Committee of the University of Hong Kong.
Free $T_{4}, T S H$, anti-TSH receptor antibody and cytokine production

These were measured as described in full previously (17). For the cytokine ELISPOT assay, which was adapted from the method of Hagiwara et al. (21), PBMC were separated within 60 minutes from blood taken into preservative-free heparin by centrifugation over Lymphoprep (Nycomed, Oslo, Norway). Multiscreen 96-well filtration plates (Millipore Corp., Bedford, MA) were used for PBMC culture. IFN$\gamma$-and IL-4-secreting cells were measured in cultures stimulated with PHA (Bacto, Detroit, MI; $10 \mu \mathrm{g} / \mathrm{mL}$ ), concanavalin A (Con A; Sigma Chemical Co., St. Louis, MO; $20 \mu \mathrm{g} / \mathrm{mL}$ ) and anti-CD3-coated Dynabeads (anti-CD3, Dynal A.S., Oslo, Norway). IL-6, IL-10, and TNF- $\alpha$-secreting cells were measured in cultures left unstimulated or stimulated with PHA, Escherichia coli 0128:B12 lipopolysaccharide (LPS; Sigma Chemical Co.; $10 \mu \mathrm{g} / \mathrm{mL}$ ) and Staphylococcus aureus Cowan I (SAC; Calbiochem, La Jolla, CA; 1:100,000). IL12-secreting cells were measured in unstimulated cultures and in cultures stimulated with PHA, anti-CD3, and SAC. All cultures were set up in duplicate and harvested after $18-22$ hours at $37^{\circ} \mathrm{C}$ in $5 \% \mathrm{CO}_{2}$.

\section{Statistical analysis}

Differences between healthy pregnant subjects and pregnant GD patients and between either group and nonpregnant controls were analyzed by Wilcoxon signed rank test. Regression analyses were by rank sum test. Prism version 2.0 software (GraphPad Software Inc., San Diego, CA) was used.
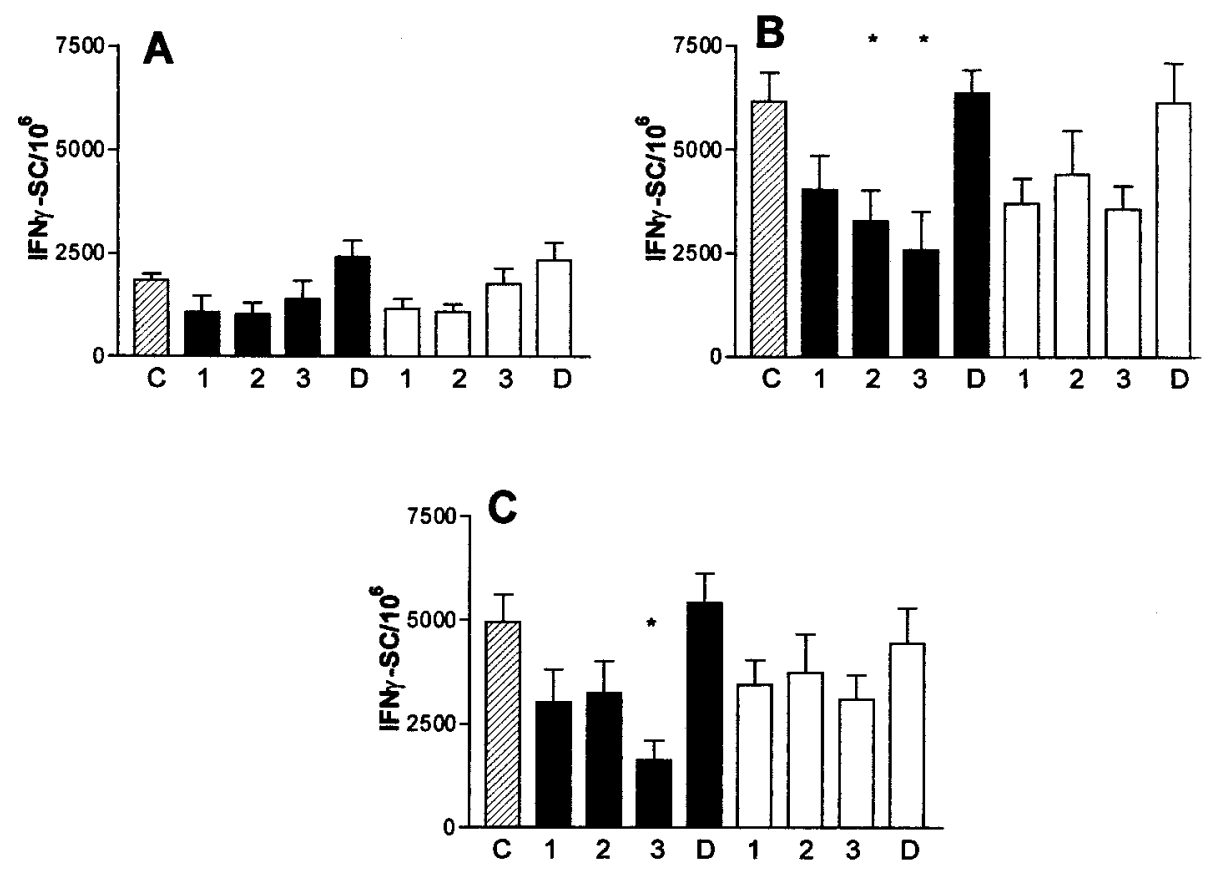

FIG. 1. Numbers of interferon- $\gamma(\mathrm{IFN}-\gamma)$-secreting cells per million peripheral blood mononuclear cells (PBMC) in enzyme-linked immunospot (ELISPOT) cultures stimulated with phytohemaglutinin (PHA) (A), concanavalin A (Con A) (B) or anti-CD3 (C). Hatched columns, normal nonpregnant controls; solid columns, pregnant controls; open columns, pregnant Graves' disease (GD) patients. 1,2,3, trimester; D, postdelivery. $* p<0.05$ cf. C, Wilcoxon signed rank test. 


\section{Results}

At first trimester, five of the patients were still positive for anti-TSH receptor antibody activity, suggesting active disease, 2 of these patients had free $\mathrm{T}_{4}$ levels above the normal range (12-23 pmol/L) and four had TSH levels below the normal range $(0.35-5.5 \mathrm{mIU} / \mathrm{L})$. Among the five patients negative for anti-TSH receptor antibody, one had free $\mathrm{T}_{4}$ above the normal range and three had suppressed TSH levels. Biochemical hyperthyroidism in these patients was presumably due to raised human chorionic gonadotrophin. Disease activity apparently moderated slightly in the second and third trimesters, with anti-TSH receptor antibody levels decreasing in all five positive patients, although one patient, who initially had low anti-TSH receptor inhibitory activity, became positive in the third trimester and remained positive 12 weeks after delivery. There was no evidence of recrudescence 10-12 weeks after delivery. All patients had normal free $\mathrm{T}_{4}$ levels by third trimester and one patient had biochemical relapse during the postpartum period.

Neither IFN- $\gamma$ - nor IL4-secreting cells are seen in unstimulated or LPS or SAC-stimulated cultures with our ELISPOT system (22). Numbers of PHA, Con A, and anti-CD3-induced IFN- $\gamma$-secreting cells were lower than control values at first, second, and third trimester in both GD and normal pregnancy, but increased to normal after delivery (Fig. 1). Numbers of T-cell activator-stimulated IL-4-secreting cells were not significantly different from control values in either normal or GD pregnancy (Fig. 2).
As shown in Figure 3, unstimulated and stimulated IL6-secreting cells were increased in normal pregnancy in the first trimester, but returned to control levels thereafter. In GD patients, IL-6 production was raised throughout pregnancy but fell to control levels after delivery. TNF- $\alpha$-secreting cells were increased in both normal and GD pregnancy and returned to normal after delivery (Fig. 4). There were significantly more PHA-stimulated TNF- $\alpha$-secreting cells in patients than controls at the second trimester $(p=0.047)$.

IL-10-secreting cells were increased in pregnancy, but with different kinetics in controls and GD (Fig. 5). Healthy pregnant women had the highest increases of IL-10 production in the first trimester, with numbers of IL-10-secreting cells falling back to control levels by the third trimester. Unstimulated and PHA-stimulated IL-10 production was increased maximally at second and third trimesters in GD pregnancy (Figs. 5A and 5B), while monocyte activators LPS and SAC induced increased IL-10 production at all three trimesters (Figs. 5C and 5D). IL-10 returned to control levels after delivery in both control and GD groups. There were significantly more LPS $(p=0.031)$ and SAC-stimulated ( $p=$ 0.031) IL-10 producing cells at third trimester in GD pregnancy than control pregnancy.

There were also marked differences in numbers of IL12-secreting cells between control and GD pregnancy (Fig. 6). IL-12 production was suppressed at second and third trimester in controls, while IL-12 was increased or normal in GD patients at first trimester and suppressed to a much smaller extent than controls at second and third trimester. Controls tended to still have lower than normal IL-12 pro-
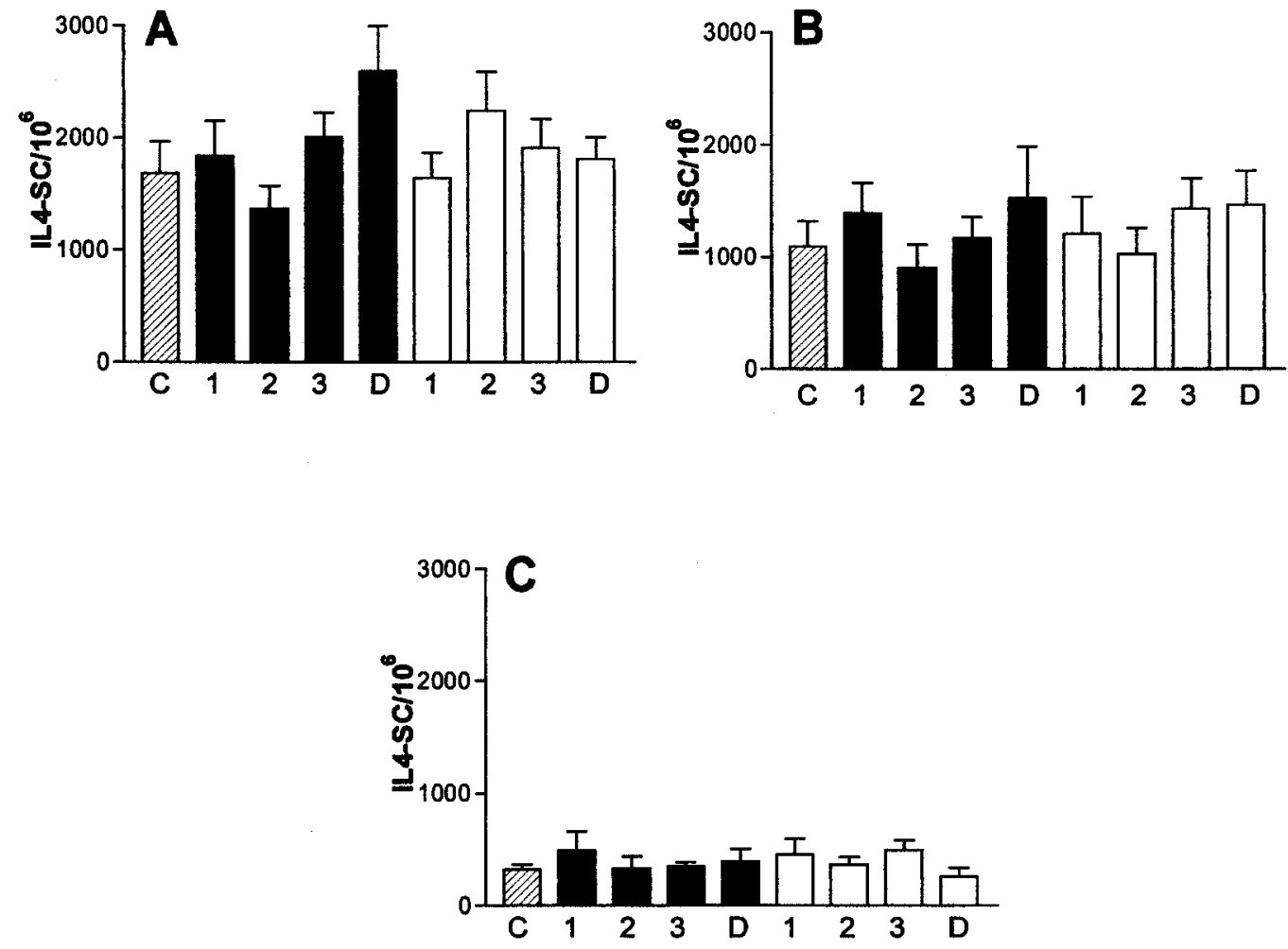

FIG. 2. Numbers of interleukin (IL)-4-secreting cells per million peripheral blood mononuclear cells (PBMC) in enzymelinked immunospot (ELISPOT) cultures stimulated with phytohemaglutinin (PHA) (A), concanavalin A (Con A) (B) or antiCD3 (C). See legend to Figure 1 for explanation of symbols and abbreviations. 

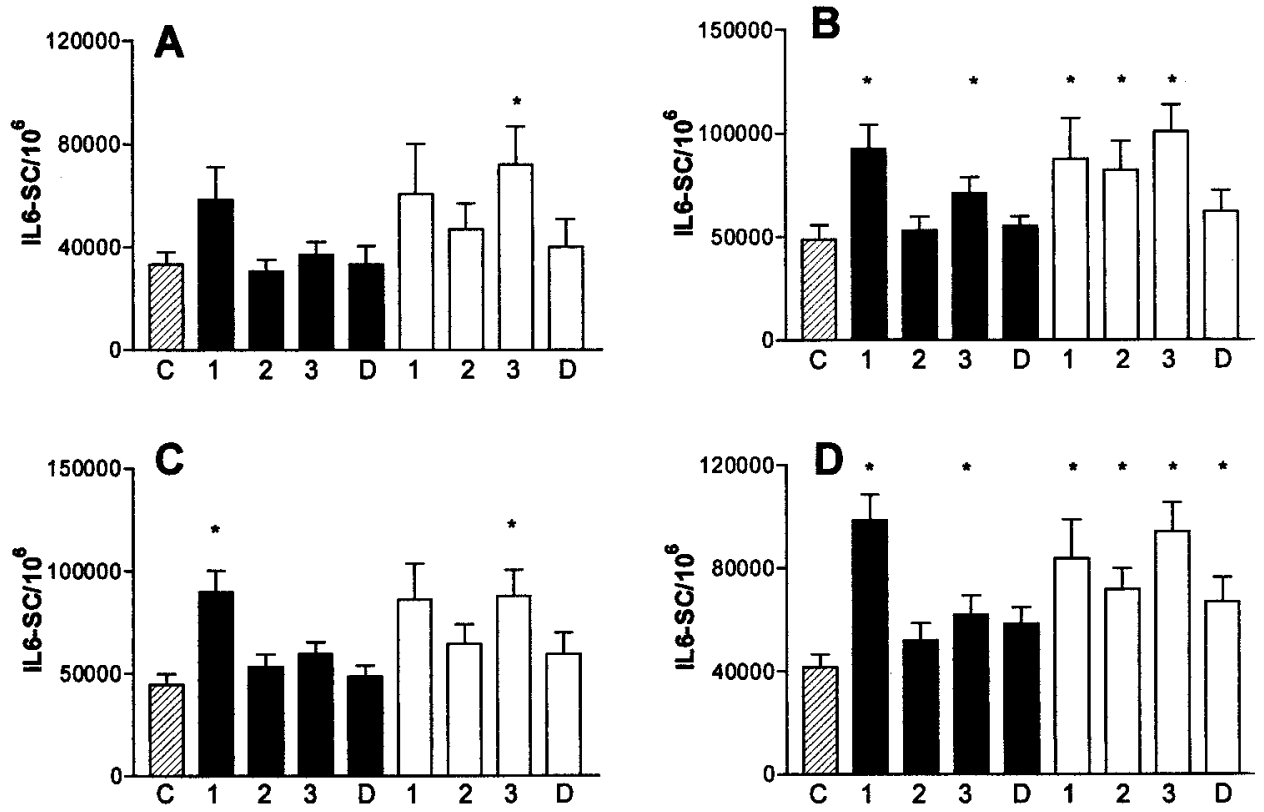

FIG. 3. Numbers of interleukin (IL)-6-secreting cells per million peripheral blood mononuclear cells (PBMC) in unstimulated enzyme-linked immunospot (ELISPOT) cultures (A) and cultures stimulated with phytohemaglutinin (PHA) (B), lipopolysaccharide (LPS) (C) or Staphylococcus aureus Cowan I (SAC) (D). See legends to Figures 1 and 2 for explanation of symbols and abbreviations.

duction at 10-12 weeks postpartum, while GD patients were essentially normal at this time.

Figure 7 shows the ratio between IL-10- and IL-12-producing cells, a measure of anti-inflammatory versus proinflammatory influences, in PHA and SAC-stimulated cul- tures. It was not possible to determine such a ratio in unstimulated cultures because the majority of subjects did not have in vivo activated IL-12-secreting cells. Ratios increased steadily during pregnancy, indicating increasingly anti-inflammatory polarization of the cytokine response. In PHA-
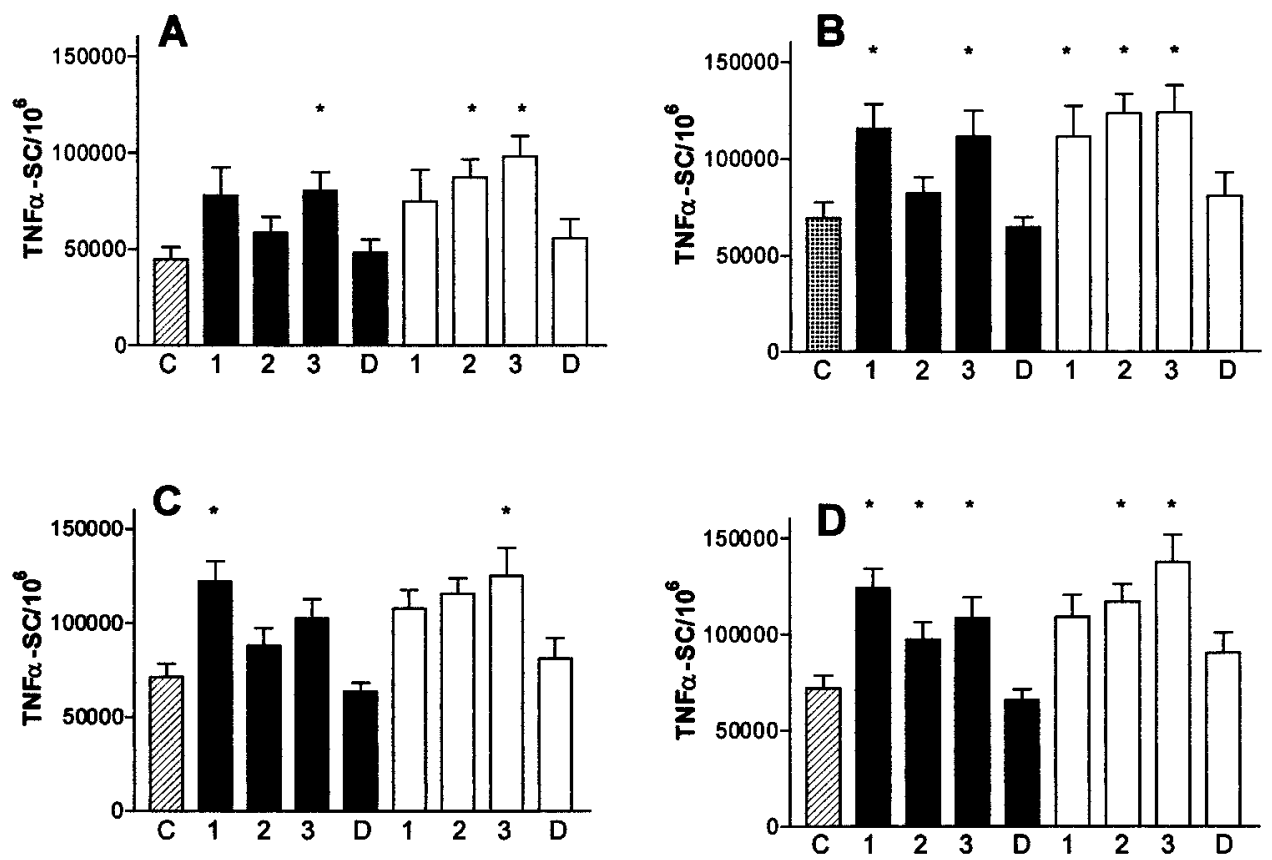

FIG. 4. Numbers of tumor necrosis factor- $\alpha$ (TNF- $\alpha$ )-secreting cells per million peripheral blood mononuclear cells (PBMC) in unstimulated enzyme-linked immunospot (ELISPOT) cultures (A) and cultures stimulated with phy tohemaglutinin (PHA) (B), lipopolysaccharide (LPS) (C) or Staphylococcus aureus Cow an I (SAC) (D). See legend to Figure 1 for explanation of symbols and abbreviations. 

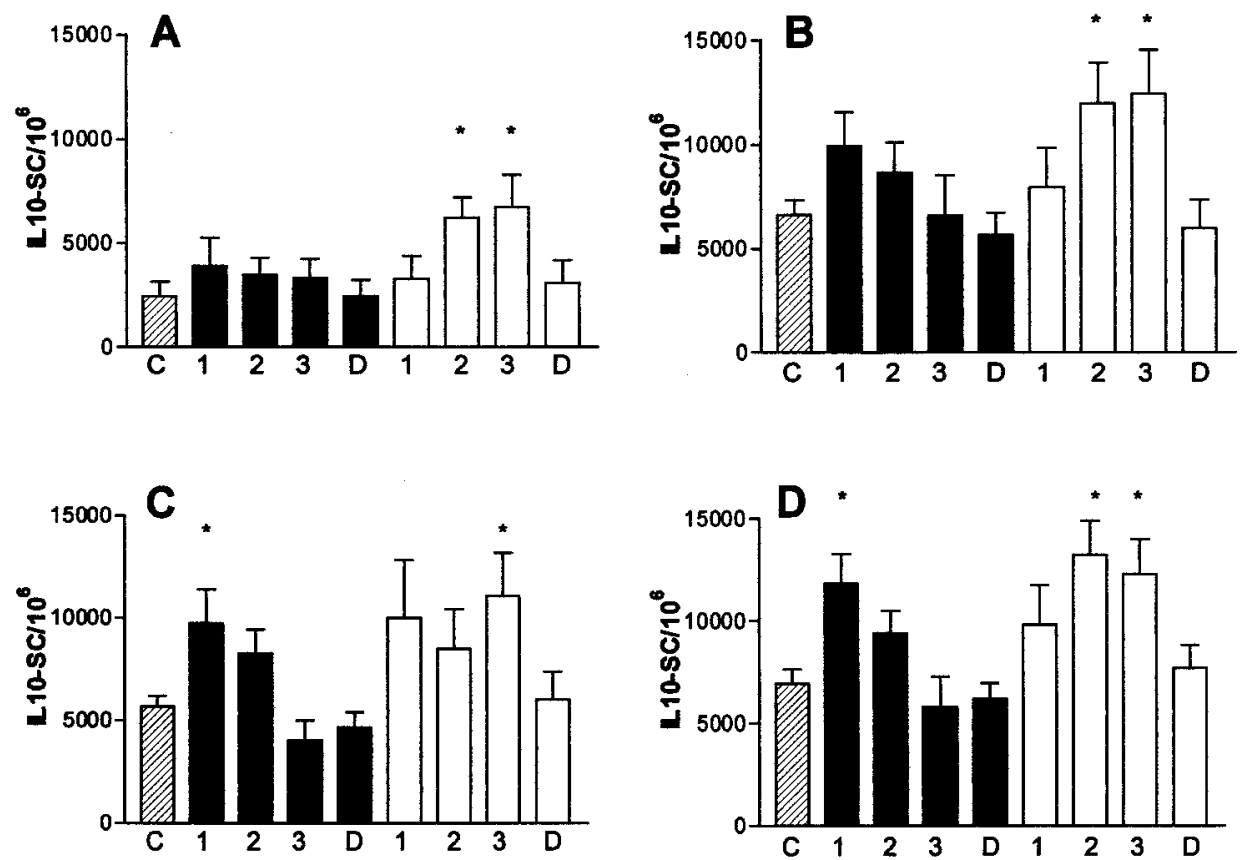

FIG. 5. Numbers of interleukin (IL)-10-secreting cells per million peripheral blood mononuclear cells (PBMC) in unstimulated enzyme-linked immunospot (ELISPOT) cultures (A) and cultures stimulated with phytohemaglutinin (PHA) (B) lipopolysaccharide (LPS) (C) or Staphylococcus aureus Cowan I (SAC) (D). See legend to Figure 1 for explanation of symbols and abbreviations.

stimulated but not SAC-stimulated cultures, pregnant GD patients achieved a far lower degree of type 2 polarization at the second and third trimester than controls. Conversely, GD patients had higher than normal SAC-induced IL-10:IL-
12 ratios at $10-12$ weeks after delivery, whereas controls returned to normal at this time.

Regression analysis failed to reveal a significant correlation between IL10:IL12 ratios and levels of anti-TSH recep-
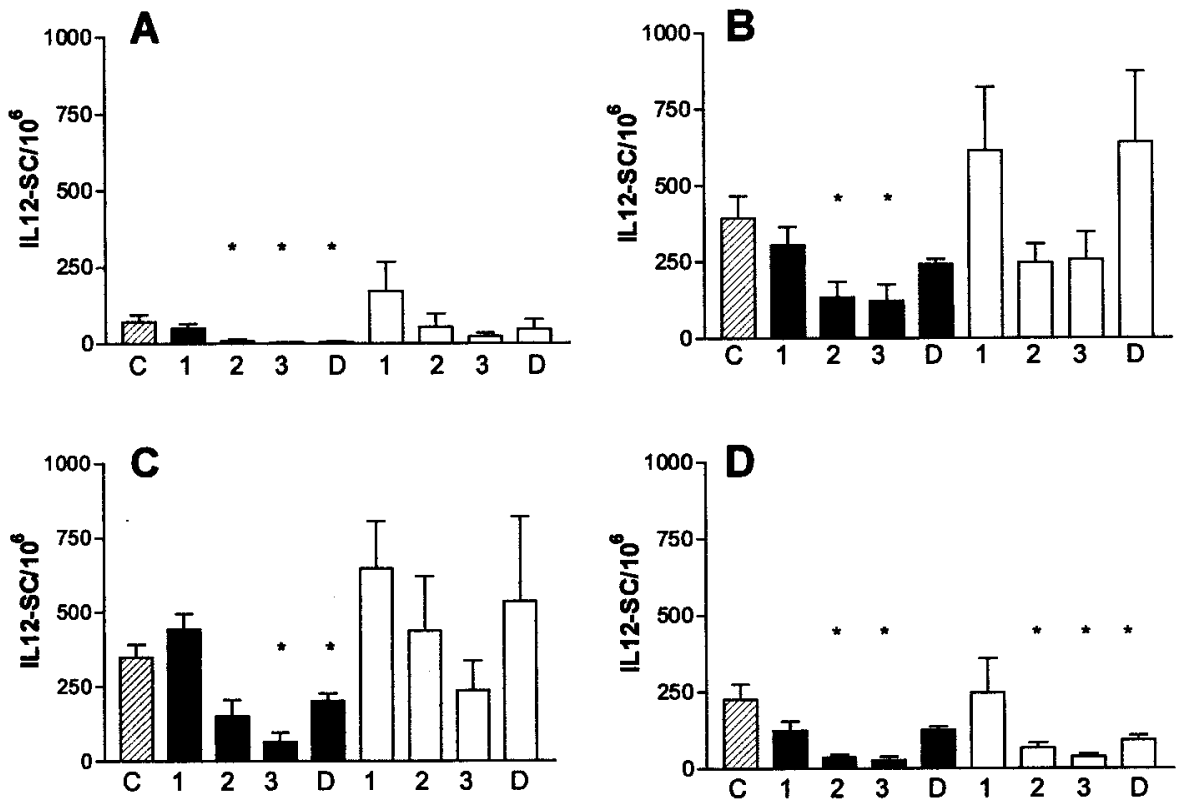

FIG. 6. Numbers of interleukin (IL)-12-secreting cells per million peripheral blood mononuclear cells (PBMC) in unstimulated enzyme-linked immunosport (ELISPOT) cultures (A) and cultures stimulated with phytohemaglutinin (PHA) (B), anti-CD3 (C), or Staphylococcus aureus Cowan I (SAC) (D). See legend to Figure 1 for explanation of symbols and abbreviations. 

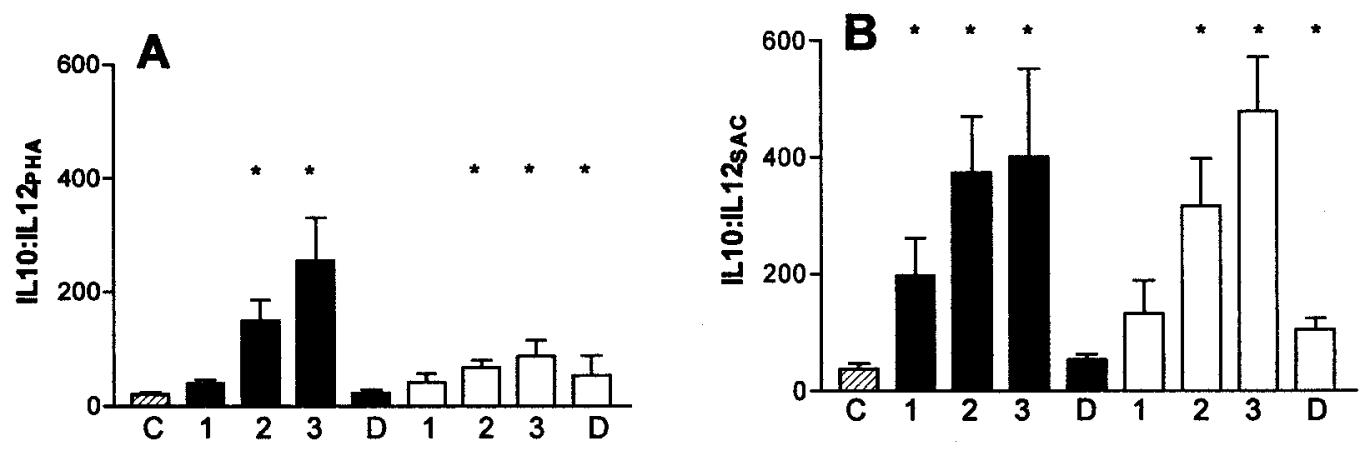

FIG. 7. Ratio of interleukin (IL)-10:IL-12-secreting cells in (A) phytohemaglutinin (PHA) and (B) Staphylococcus aureus Cowan I (SAC)-stimulated cultures. See legend to Figure 1 for explanation of symbols and abbreviations.

tor inhibitory antibody (Pearson $r=0.2801, p=0.080)$, TSH (Pearson $r=0.2068, p=0.20$ ) or free $\mathrm{T}_{4}$ (Pearson $r=0.0051$, $p=0.975$.).

\section{Discussion}

The study of cytokine interactions in women with autoimmune diseases who have become pregnant provides a unique opportunity to examine hormonal and immunologic influences on disease expression and greater understanding of these interactions should yield clues to novel therapeutic manipulations. In the present study there were both similarities and differences in cytokine production between normal and GD pregnancy.

Normal pregnancy is accompanied by decreased production of type 1 proinflammatory cytokines and increased production of type 2 anti-inflammatory cytokines $(1,2)$. Antigenand mitogen-stimulated IL-2 and IFN- $\gamma$ were suppressed and IL-4 and IL-10 increased in PBMC culture supernatants, with polarization toward type 2 cytokines increasing to maximum at the third trimester (23). The results presented here indicate somewhat different kinetics when numbers of cytokine-secreting cells are evaluated by ELISPOT: enhancement of IL-10 production occurred in the first trimester of normal pregnancy and decreased thereafter. Furthermore, changes in numbers of IL-4-producing cells were relatively minor in our study. IL-12 was suppressed to a greater extent as pregnancy advanced and had not recovered to normal levels even by 11 weeks after delivery. IFN- $\gamma$ was suppressed throughout pregnancy, with different kinetics depending on the nature of the activating stimulus. Our data suggests that increased IL-10 and decreased IFN- $\gamma$ are early events in normal pregnancy and suppression of IL-12 occurs later and is self-sustaining even when IL-10 production returns to normal.

In the present study, IL-6 was found to be elevated at first trimester and TNF- $\alpha$ was increased throughout pregnancy in controls. This is in contrast to a previous study showing no significant changes in plasma levels of IL-6 and TNF- $\alpha$ during normal pregnancy, but considerable increases associated with preeclampsia (24). Differences in findings are probably accounted for by different methodologies, in particular the susceptibility of measurements in plasma to inhibitory influences such as receptor binding (25).

Pregnant GD patients generally had similar changes in numbers of IFN- $\gamma$ and IL-4-producing cells as control pregnant women. IL-6 production was increased in patients but not controls at second and third trimester and TNF- $\alpha$ production was higher in patients than controls at the second trimester, when controls but not patients temporarily became normal. These latter findings might indicate increased inflammatory activity during pregnancy in GD.

The clearest differences between normal and GD pregnancy were in IL-10 and IL-12-secreting cells. IL-10 was increased throughout pregnancy in GD patients but returned to normal by third trimester in controls. IL-12 was suppressed to a much lesser extent at all times in GD pregnancy. Because IL-10 increases preceded IL-12 decreases in normal pregnancy it would appear that failure to suppress IL-12 production in pregnant GD patients is because of poor responses to apparently adequate IL-10 levels, which may have been sustained for longer than normal because of failure to downregulate $\mathrm{IL}-12$.

Ratios of IL-10:IL-12-secreting cells clearly showed deficient type 2 cytokine polarization in GD patients at second and third trimester in PHA-stimulated cultures, but was less apparent using SAC activation. SAC directly stimulates IL12 production by monocytes, whereas PHA induces T-cells to express CD154 that then induces IL-12 production by monocytes via CD40 ligation (26). The latter pathway is clearly not responsive to regulation by IL-10 in GD pregnancy, while the direct pathway can apparently respond normally.

Cytokine profiles of GD patients 10-12 weeks after delivery were essentially normal, which is in contrast to our previous study of patients before and after radioiodine therapy, whose IL-4 and IFN- $\gamma$ production was initially deficient (17). The pregnant patients, however, had mostly achieved disease remission prior to conception, whereas the previously studied patients required radiotherapy for newly diagnosed disease or disease not adequately controlled by drugs. Despite the differences in clinical features of the two groups, there was also evidence from the first study that regulation of IL-12 production by IL-10 might be deficient, because IL10 production was greatly increased 17 days after radioactive iodine, but there was no concomitant decrease in IL-12.

Small numbers of subjects were studied here and only one subject had highly active disease. The finding of apparently aberrant regulation of IL-12 by IL-10 in GD should be confirmed in larger studies including more active patients. 


\section{Acknowledgments}

This work was supported by the Committee for Research and Conference Grants, University of Hong Kong. We are indebted to our patients for their willing cooperation.

\section{References}

1. Wegmann TG, Lin, H, Guilbert L, Mosmann TR 1993 Bidirectional cytokine interactions in the maternal-fetal relationship: is successful pregnancy a $\mathrm{T}_{\mathrm{H}} 2$ phenomenon? Immunol Today 14:353-356.

2. Lin H, Mosmann TR, Guilbert L, Tuntipotat S, Wegmann TG 1993 Synthesis of T helper 2-type cytokines at the maternalfetal interface. J Immunol 151:4562-4573.

3. Elenkof IJ, Papanicolaou DA, Wilder RL, Chrousos GP 1996 Modulatory effects of glucocorticoids and catecholamines on human interleukin-12 and interleukin-10 production: clinical implications. Proc Assoc Am Physicians 108:374-381.

4. Gilmore W, Weiner L, Correale J 1997 Effect of estradiol on cytokine secretion by proteolipid protein-specific T-cell clones isolated from multiple sclerosis patients and normal control subjects. J Immunol 158:446-451.

5. Piccinni MP, Giudizi MG, Biagiotti R, Beloni L, Giannarini L, Sampognaro S, Parronchi P, Manetti R, Annunziato F, Livi C, et al 1995 Progesterone favours the development of human T-helper cells producing Th2-type cytokines and promotes both IL-4 production and membrane CD30 expression in established Th1 cell clones. J Immunol 155:128-133.

6. Feldmann M. Brennan FM, Maini RN 1996 Role of cytokines in rheumatoid arthritis. Annu Rev Immunol 14:397-440.

7. Oka M, Vaino U 1966 Effect of pregnancy on the prognosis and serology of rheumatoid arthritis. Acta Rheum Scand 12:47-52.

8. Wilder RL 1998 Hormones, pregnancy and autoimmune diseases. Ann N Y Acad Sci 840:45-50.

9. Buyon JP, Nelson JL, Lockshin MD 1996 The effects of pregnancy on autoimmune diseases. Clin Immunol Immunopathol 78:99-104.

10. Paschke R, Schuppert F, Taton M, Velu T 1994 Intrathyroidal cytokine gene expression profiles in autoimmune thyroiditis. J Endocrinol 141:309-315.

11. Roura-Mir C, Catalfamo M, Sospedra M, Alcade L, PujolBorrell R, Jaraquemada D 1997 Single cell analysis of intrathyroidal lymphocytes shows differential cytokine expression in Hashimoto's and Graves' disease. Eur J Immunol 12:3290-3302.

12. Watson PF, Pickeril AP, Davies R, Weetman AP 1994 Analysis of cytokine gene expression in Graves' disease and multinodular goitre. J Clin Endocrinol Metab 79:355-360.

13. Heuer M, Aust G, Ode-Hakim S, Scherbaum WA 1996 Different cytokine profiles in Graves' disease, Hashimoto's thyroiditis and non-autoimmune thyroid disorders determined by quantitative reverse transcriptase polymerase chain reaction (RT-PCR). Thyroid 6:97-106.

14. Guo J, Rapoport B, McLachlan SM 1997 Cytokine profiles of in vivo activated thyroid infiltrating $\mathrm{T}$-cells cloned in the presence or absence of interleukin 4. Autoimmunity 26: 103-110.

15. Hirooka Y, Kaynmara M, Ohga S, Kimura M, Hasegawa M, Shin K, Nogimori T, Ishizuki Y, Mitsuma T 1993 Deregu- lated production of interleukin-4 (IL4) in autoimmune thyroid disease assayed with a new radioimmunoassay. Clin Chim Acta 216:1-10.

16. Kallman BA, Hurther M, Tuber M, Feldkamp J, Bertrams J, Gries FA, Lampeter EF, Kolb H 1997 Systemic bias of cytokine production towards cell-mediated immune regulation in IDDM and towards humoral immunity in Graves' disease. Diabetes 46:237-243.

17. Jones BM, Kwok CCH, Kung, AWC 1999 Effect of radioactive iodine therapy on cytokine production in Graves' disease: Transient increases in interleukin-4 (IL-4), IL-6, IL-10, and tumour necrosis factor- $\alpha$, with longer term increases in interferon- $\gamma$ production. J Clin Endocrinol Metabol 84:41064110.

18. McLachlan SM, Taverne J, Atherton MC, Cooke A, Middleton S, Pegg CA, Clark F, Rees Smith B 1990 Cytokines, thyroid autoantibody synthesis and thyroid cell survival in culture. Clin Exp Immunol 79:175-181.

19. Amino N, Tanizawa O, Mori H, et al 1982 Aggravation of thyrotoxicosis in early pregnancy and after delivery in Graves' disease. J Clin Endocrinol Metab 55:108-112.

20. Kung AWC, Jones BM 1998 A change from stimulatory to blocking antibody activity in Graves' disease during pregnancy. J Clin Endocrinol Metabol 83:514-518.

21. Hagiwara E, Gourley MF, Lee S, Klinman DM 1996 Disease severity in patients with systemic lupus erythematosus correlates with an increased ratio of interleukin-10:interferon$\gamma$-secreting cells in peripheral blood. Arthritis Rheum 39:379-385.

22. Jones BM, Liu TF, Wong RWS 2000 Reduced in vitro production of interferon-gamma, interleukin-4 and interleukin12 and increased production of interleukin-6, interleukin-10 and tumour necrosis factor-alpha in systemic lupus erythematosus. Weak correlations with disease activity. Autoimmunity 31:117-124.

23. Marzi M, Vigano A, Trabattoni D, Villa ML, Salvaggio A, Clerici E, Clerici M 1996 Characterization of type 1 and type 2 cytokine production profile in physiologic and pathologic human pregnancy. Clin Exp Immunol 106:127-133.

24. Conrad KP, Miles TM, Benyo DF 1998 Circulating levels of immunoreactive cytokines in women with preeclampsia. Am J Reprod Immunol 40:102-111.

25. Kapadia S, Torre-Amione G, Mann DL 1994 Pitfalls in measuring cytokines. Ann Intern Med 121:149-150.

26. Maruo S, Oh-hora M, Ahn HJ, Ono S, Wysocka M, Kaneko Y, Yagita H, Okumura K, Kikutani H, Kishimoto T, Kobayashi M, Hamaoka T, Trinchieri G, Fujiwara H 1997 Bcells regulate CD40 ligand-induced IL-12 production in antigen-presenting cells (APC) during T-cell/ APC interactions. J Immunol 158:120-126.

Address reprint requests to: Dr. B. M. Jones Division of Clinical Immunology Department of Pathology Queen Mary Hospital Pokfulam Hong Kong

E-mail: bmjones@ha.org.uk 